\title{
A lower bound for the error term in Weyl's law for certain Heisenberg manifolds, II
}

\author{
Werner Georg Nowak ${ }^{*}$ (Vienna)
}

\begin{abstract}
This article is concerned with estimations from below for the remainder term in Weyl's law for the spectral counting function of certain rational $(2 \ell+1)$-dimensional Heisenberg manifolds. Concentrating on the case of odd $\ell$, it continues the work done in part I [20] which dealt with even $\ell$.
\end{abstract}

1. Introduction. Weyl's law and Heisenberg manifolds. Let $M$ be a closed $n$-dimensional Riemannian manifold with a metric $g$ and Laplace-Beltrami operator $\Delta$. Denote by $N(t)$ the spectral counting function

$$
N(t):=\sum_{\substack{\text { eigenvalue of } \Delta \\ \lambda \leq t}} d(\lambda)
$$

where $d(\lambda)$ is the dimension of the eigenspace corresponding to $\lambda$, and $t$ is a large real variable. According to a deep general theorem of L. Hörmander [10],

$$
N(t)=\frac{\operatorname{vol}(M)}{(4 \pi)^{n / 2} \Gamma\left(\frac{1}{2} n+1\right)} t^{n / 2}+O\left(t^{(n-1) / 2}\right),
$$

where the error term - in this general setting - is best possible. Asymptotics like (1.1) and its refinements for special manifolds are usually subsumed under the notion of Weyl's law.

Recently, the spectral theory of so-called Heisenberg manifolds has attracted a lot of attention, possibly on the grounds of motivation from quantum physics and the abstract theory of PDE's. To recall basics, let $\ell \geq 1$ be an integer, and put ${ }^{(1)}$

$$
\gamma(\mathbf{x}, \mathbf{y}, z)=\left(\begin{array}{ccc}
1 & \mathbf{x} & z \\
{ }^{t} \mathbf{o}_{\ell} & I_{\ell} & { }^{t} \mathbf{y} \\
0 & \mathbf{o}_{\ell} & 1
\end{array}\right)
$$

Mathematics Subject Classification (2000): 11N37, 35P20, 58J50, 11P21.

(*) The author gratefully acknowledges support from the Austrian Science Fund (FWF) under project Nr. P20847-N18.

(1) Bold face letters will denote throughout elements of some space $\mathbb{R}^{n}$, resp., of $\mathbb{Z}^{n}$. They may be viewed also as $(1 \times n)$-matrices ("row vectors") where applicable. 
where $\mathbf{x}, \mathbf{y} \in \mathbb{R}^{\ell}, z \in \mathbb{R}, \mathbf{o}_{\ell}=(0, \ldots, 0) \in \mathbb{R}^{\ell}, I_{\ell}$ is the $(\ell \times \ell)$-unit matrix, and ${ }^{t}$. denotes transposition. Then the $(2 \ell+1)$-dimensional Heisenberg group $H_{\ell}$ is defined by

$$
H_{\ell}=\left\{\gamma(\mathbf{x}, \mathbf{y}, z): \mathbf{x}, \mathbf{y} \in \mathbb{R}^{\ell}, z \in \mathbb{R}\right\}
$$

with the usual matrix product. Further, for any $\ell$-tuple $\mathbf{r}=\left(r_{1}, \ldots, r_{\ell}\right) \in \mathbb{Z}_{+}^{\ell}$ with the property that $r_{j} \mid r_{j+1}$ for all $j=1, \ldots, \ell-1$, let $\mathbf{r} * \mathbb{Z}^{\ell}:=r_{1} \mathbb{Z} \times \ldots \times r_{\ell} \mathbb{Z}$, and define

$$
\Gamma_{\mathbf{r}}=\left\{\gamma(\mathbf{x}, \mathbf{y}, z): \mathbf{x} \in \mathbf{r} * \mathbb{Z}^{\ell}, \mathbf{y} \in \mathbb{Z}^{\ell}, z \in \mathbb{Z}\right\}
$$

$\Gamma_{\mathbf{r}}$ is a uniform discrete subgroup of $H_{\ell}$, i.e., the Heisenberg manifold $H_{\ell} / \Gamma_{\mathbf{r}}$ is compact. According to Gordon and Wilson [6], Theorem 2.4, the subgroups $\Gamma_{\mathbf{r}}$ classify all uniform discrete subgroups of $H_{\ell}$ up to automorphisms: For every uniform discrete subgroup $\Gamma$ of $H_{\ell}$ there exists a unique $\ell$-tuple $\mathbf{r}$ and an automorphism of $H_{\ell}$ which maps $\Gamma$ to $\Gamma_{\mathbf{r}}$. However, to get a "rational" or "arithmetic" Heisenberg manifold - this latter expression being due to Petridis \& Toth [21] - one has to make a quite particular choice of the metric $^{(2)} g$. Following Petridis \& Toth [21], Theorem 1.1, and also Zhai [24], we choose

$$
g_{\ell}=\left(\begin{array}{cc}
I_{2 \ell} & { }^{t} \mathbf{o}_{2 \ell} \\
\mathbf{o}_{2 \ell} & 2 \pi
\end{array}\right) .
$$

The spectrum of the Laplace-Beltrami operator on $\left(H_{\ell} / \Gamma_{\mathbf{r}}, g_{\ell}\right)$ has been analyzed in Gordon and Wilson [6], p. 259, and also in Khosravi and Petridis [16], p. 3564. It consists of two different classes $\mathcal{S}_{I}$ and $\mathcal{S}_{I I}$, where $\mathcal{S}_{I}$ is the spectrum of the Laplacian on the $2 \ell$-dimensional torus $\mathbb{R}^{2 \ell} / \mathbb{Z}^{2 \ell}$, and

$$
\mathcal{S}_{I I}=\left\{2 \pi\left(n_{0}^{2}+n_{0}\left(2 n_{1}+\ell\right)\right): n_{0} \in \mathbb{Z}^{+}, n_{1} \in \mathbb{Z}_{0}^{+}\right\}
$$

with multiplicities ( $=$ dimensions of corresponding eigenspaces) $2 n_{0}^{\ell} r_{1} \cdot \ldots \cdot r_{\ell}\left(\begin{array}{c}n_{1}+\ell-1 \\ \ell-1\end{array}\right)$.

\section{Lattice points in a circle. The quantity}

$$
\sum_{\substack{\lambda \in \mathcal{S}_{I I} \\ \lambda \leq t}} d(\lambda)
$$

yields the major contribution to $N(t)$ for this rational Heisenberg manifolds. Its asymptotic evaluation amounts to the enumeration of the integer points $\left(n_{0}, n_{1}\right) \in\left(\mathbb{Z}_{0}^{+}, \mathbb{Z}^{+}\right)$ in the planar domain $u^{2}+u(2 v+\ell) \leq t /(2 \pi)$, with the weights $2 n_{0}^{\ell} r_{1} \cdot \ldots \cdot r_{\ell}\left(\begin{array}{c}n_{1}+\ell-1 \\ \ell-1\end{array}\right)$ as indicated. This observation may be considered as one motivation to make reference to the state-of-art with the Gaussian circle problem, the "ancestor and prototype" of all planar lattice point problems. As a second link, one may notice that $M=\mathbb{R}^{2} / \mathbb{Z}^{2}$, the 2-dimensional torus, is the simplest example of a Riemannian manifold with a non-trivial

(2) Compare the discussion below concerning the bound (3.3) which applies to "almost all" metrics $g$. 
spectral theory: In fact ${ }^{(3)}$, the eigenvalues of the Laplacian on $\mathbb{R}^{2} / \mathbb{Z}^{2}$ are given by $4 \pi^{2} k$, where $k$ ranges over all nonnegative integers with $r(k)>0, r(k)$ denoting as usual the number of ways to write $k$ as a sum of two squares of integers. The corresponding multiplicities are given by $r(k)$, hence the spectral counting function $N(t)$ now equals the number of lattice points in an origin-centered compact circular disc of radius $\sqrt{t} /(2 \pi)$.

For enlightening accounts on the history of the Gaussian circle problem in textbook style, the reader may consult the monographs of Krätzel [18], [19], and Huxley [11], along with the recent quite comprehensive survey article [13]. The sharpest upper bound for the lattice point discrepancy $P(x)$ of the compact unit circular disc $\mathcal{D}_{0}$, linearly dilated by a large real parameter $x$, is nowadays due to Huxley [12] and reads

$$
P(x):=\#\left(x \mathcal{D}_{0} \cap \mathbb{Z}^{2}\right)-\pi x^{2}=O\left(x^{131 / 208}(\log x)^{18637 / 8320}\right) .
$$

It is usually conjectured that $P(x)=O\left(x^{1 / 2+\varepsilon}\right)$ for every $\varepsilon>0$. This is supported by Cramér's [3] classic mean-square asymptotics

$$
\int_{0}^{X}(P(x))^{2} \mathrm{~d} x \sim C X^{2}
$$

with an explicit constant $C>0$. Thus, roughly speaking, $P(x) \ll x^{1 / 2}$ in square-mean, but it has been known for a long time that there exist unbounded sequences of $x$-values for which $P(x)$ attains "exceptionally large" values, even of either sign: By 1961, the state-of-art in this direction was that ${ }^{(4)}$

$$
P(x)=\Omega_{-}\left(x^{1 / 2}(\log x)^{1 / 4}\right)
$$

and

$$
P(x)=\Omega_{+}\left(x^{1 / 2}\left(\log _{2} x \log _{3} x\right)^{1 / 4}\right)
$$

due to Hardy [9], resp., Gangadharan [5]. Here and throughout, $\log _{j}$ stands for the $j$-fold iterated logarithm. Later on, these estimates have been improved: Corrádi and Kátai [2] obtained

$$
P(x)=\Omega_{+}\left(x^{1 / 2} \exp \left(C_{1}\left(\log _{2} x\right)^{1 / 4}\left(\log _{3} x\right)^{-3 / 4}\right)\right),
$$

Hafner [8] established

$$
P(x)=\Omega_{-}\left(x^{1 / 2}(\log x)^{1 / 4}\left(\log _{2} x\right)^{(\log 2) / 4} \exp \left(-C_{2}\left(\log _{3} x\right)^{1 / 2}\right)\right),
$$

(3) See also the detailed discussion in part I of this work [20].

(4) Recall the usual $\Omega$-notation: For real functions $F$ and $G>0$, and * denoting either + or - , $F(x)=\Omega_{*}(G(x))$ means that $\lim \sup (* F(x) / G(x))>0$, as $x \rightarrow \infty$. Further, $F(x)=\Omega(G(x))$ means that $\lim \sup |F(x) / G(x)|>0$. 
and Soundararajan [22] proved that

$$
P(x)=\Omega\left(x^{1 / 2}(\log x)^{1 / 4}\left(\log _{2} x\right)^{3\left(2^{1 / 3}-1\right) / 4}\left(\log _{3} x\right)^{-5 / 8}\right) .
$$

The bounds (2.5) - (2.7) depend on the special multiplicative structure of the arithmetic function $r(n)$, and on the analytic properties of its generating Dirichlet series (Epstein zeta-function).

3. Results on the spectral counting function of Heisenberg manifolds. Returning to rational Heisenberg manifolds $M=\left(H_{\ell} / \Gamma_{\mathbf{r}}, g_{\ell}\right)$ as described in section 1, we give an account of what is known about the error term in (1.1), i.e.,

$$
R(t)=N(t)-\frac{\operatorname{vol}(M)}{(4 \pi)^{\ell+1 / 2} \Gamma\left(\ell+\frac{3}{2}\right)} t^{\ell+1 / 2}=N(t)-\frac{r_{1} \cdot \ldots \cdot r_{\ell}}{2^{2 \ell+1 / 2} \pi^{\ell} \Gamma\left(\ell+\frac{3}{2}\right)} t^{\ell+1 / 2} .
$$

For $\ell=1$, Petridis and Toth [21] proved that $R(t) \ll t^{5 / 6} \log t$. This estimate was sharpened and generalized to arbitrary $\ell \geq 1$ by Khosravi and Petridis [16] who established $R(t) \ll t^{\ell-7 / 41}$. In a recent paper, Zhai [24] applied Huxley's "discrete Hardy-Littlewood method" [11], [12] to derive, for any $\ell \geq 1$,

$$
R(t) \ll t^{\ell-77 / 416}(\log t)^{26957 / 8320} .
$$

Actually, it is just the special "rational" choice of the metric $g_{\ell}$ which makes the error term (possibly) large. As Khosravi and Petridis [16] showed, for "almost all" metrics $g$, the much sharper bound

$$
R_{g}(t) \ll_{g} t^{\ell-1 / 4} \log t
$$

holds true. Returning to the rational case (1.4), a result of Khosravi [15] and Khosravi \& Toth [17] tells us that

$$
\int_{0}^{T}(R(t))^{2} \mathrm{~d} t=C_{\ell} T^{2 \ell+1 / 2}+O\left(T^{2 \ell+1 / 4+\varepsilon}\right)
$$

where $C_{\ell}>0$ is an explicit constant. A recent paper of Zhai [24] is concerned with estimates and asymptotics for higher power moments of $R(t)$. In fact, (3.3) and (3.4) may suggest the conjecture that

$$
R(t) \ll t^{\ell-1 / 4+\varepsilon}
$$

for every $\varepsilon>0$. The results described so far show a lot of analogy to the Gaussian circle problem discussed in section 2 .

In the present work, it is our objective to estimate $R(t)$ from below, in order to arrive again at a statement saying that " $R(t) \ll t^{\ell-1 / 4}$ in mean-square, with an unbounded 
sequence of exceptionally large values $t$ ". In fact, we are able to find for each $\ell \geq 1$ an explicit function $\omega_{\ell}(t)$ tending to $\infty$, such that

$$
R(t)=\Omega\left(t^{\ell-1 / 4} \omega_{\ell}(t)\right) .
$$

Theorem. For any fixed positive integer $\ell$, let $\left(H_{\ell} / \Gamma_{\mathbf{r}}, g_{\ell}\right)$ be a rational $(2 \ell+1)$ dimensional Heisenberg manifold with metric $g_{\ell}$, as described above. Then the error term $R(t)$ for the associated spectral counting function, defined in (3.1), satisfies

$$
\limsup _{t \rightarrow \infty} \frac{R(t)}{t^{\ell-1 / 4} \omega_{\ell}(t)}>0
$$

where

$$
\omega_{\ell}(t):= \begin{cases}(\log t)^{1 / 4} & \text { for } \ell \text { even } \\ \left(\log _{2} t \log _{3} t\right)^{1 / 4} & \text { for } \ell \text { odd }\end{cases}
$$

Remarks. 1. The case of even $\ell$ has been treated in the first part of this work [20]. After approximating $R(t)$ by a suitable trigonometric sum, the Dirichlet approximation theorem was applied to give all its terms the positive sign. In the present article we shall deal with the case of odd $\ell$, employing a quantitative version of Kronecker's theorem instead. Technically, this will be stated in terms of uniform distribution theory - see Lemma 4 below.

2. Our results obviously are comparable to the bounds (2.3) and (2.4) for the circle problem. It seems very difficult to obtain improvements as sharp as (2.5) - (2.7), since the coefficients $\theta_{\ell}(n)$ (defined in (5.11) below) fail to share the useful properties of $r(n)$.

3. For the circle problem, the two different types of arguments (Dirichlet's theorem vs. Kronecker's) were used to establish $\Omega_{-}$- and $\Omega_{+}$-results. For Heisenberg manifolds they are needed to deal with $\ell$ of arbitrary parity, yielding $\Omega_{+}$-bounds in both cases.

\section{Some Lemmas.}

Lemma 1. (Vaaler's approximation of fractional parts by trigonometric polynomials.) For arbitrary $w \in \mathbb{R}$ and $H \in \mathbb{Z}^{+}$, let $\psi(w):=w-[w]-\frac{1}{2}$,

$$
\Sigma_{H}(w):=\sum_{h=1}^{H} \alpha_{h, H} \sin (2 \pi h w), \quad \Sigma_{H}^{*}(w):=\sum_{h=1}^{H} \beta_{h, H} \cos (2 \pi h w)+\frac{1}{2 H+2},
$$

where, for $h=1, \ldots, H$,

$$
\alpha_{h, H}:=\frac{1}{\pi h} \rho\left(\frac{h}{H+1}\right), \quad \beta_{h, H}:=\frac{1}{H+1}\left(1-\frac{h}{H+1}\right)
$$


and

$$
\rho(\xi)=\pi \xi(1-\xi) \cot (\pi \xi)+\xi \quad(0<\xi<1) .
$$

Then the following inequality holds true:

$$
\left|\psi(w)+\Sigma_{H}(w)\right| \leq \Sigma_{H}^{*}(w) .
$$

Proof. This is one of the main results in Vaaler [23]. A very well readable exposition can also be found in the monograph by Graham and Kolesnik [7].

Lemma 2. Let $F \in C^{4}[A, B], G \in C^{2}[A, B]$, and suppose that, for positive parameters $X, Y, Z$, we have $1 \ll B-A \ll X$ and

$$
F^{(j)} \ll X^{2-j} Y^{-1} \quad \text { for } j=2,3,4,\left|F^{\prime \prime}\right| \geq c_{0} Y^{-1}, \quad G^{(j)} \ll X^{-j} Z \quad \text { for } j=0,1,2,
$$

throughout the interval $[A, B]$, with some constant $c_{0}>0$. Let $\mathcal{J}^{\prime}$ denote the image of ]$A, B]$ under $F^{\prime}$, and $F^{*}$ the inverse function of $F^{\prime}$. Then, with $e(w)=e^{2 \pi i w}$ as usual,

$$
\begin{aligned}
\sum_{A<m \leq B} G(m) e(F(m))= & e\left(\frac{\operatorname{sgn}\left(F^{\prime \prime}\right)}{8}\right) \sum_{k \in \mathcal{J}^{\prime}} \frac{G\left(F^{*}(k)\right)}{\sqrt{\left|F^{\prime \prime}\left(F^{*}(k)\right)\right|}} e\left(F\left(F^{*}(k)\right)-k F^{*}(k)\right)+ \\
& +O\left(Z\left(\sqrt{Y}+\log \left(2+\operatorname{length}\left(\mathcal{J}^{\prime}\right)\right)\right)\right) .
\end{aligned}
$$

Proof. Transformation formulas of this kind are quite common, though often with worse error terms. This very sharp version can be found as f. (8.47) in the recent monograph [14] of H. Iwaniec and E. Kowalski.

Lemma 3. For a real parameter $T \geq 1$, let $\mathcal{F}_{T}$ denote the Fejér kernel

$$
\mathcal{F}_{T}(v)=T\left(\frac{\sin (\pi T v)}{\pi T v}\right)^{2} .
$$

Then for arbitrary real numbers $Q>0$ and $\delta$, it follows that

$$
\int_{-1}^{1} \mathcal{F}_{T}(v) e(Q v+\delta) \mathrm{d} v=\max \left(1-\frac{Q}{T}, 0\right) e(\delta)+O\left(\frac{1}{Q}\right),
$$

where the $O$-constant is independent of $T$ and $\delta$.

Proof. This useful result is due to Hafner [8]. It follows from the classic Fourier transform formula

$$
\int_{\mathbb{R}} \mathcal{F}_{T}(v) e(Q v) \mathrm{d} v=\int_{\mathbb{R}}\left(\frac{\sin (\pi v)}{\pi v}\right)^{2} e\left(\frac{Q}{T} v\right) \mathrm{d} v=\max \left(1-\frac{Q}{T}, 0\right) .
$$


Since $\mathcal{F}_{T}( \pm 1) \ll T^{-1}$ and $\mathcal{F}_{T}^{\prime}(v) \ll v^{-2}$ for $|v| \geq 1$, uniformly in $T \geq 1$, integration by parts readily shows that the intervals $]-\infty,-1]$ and $\left[1, \infty\left[\right.\right.$ contribute only $O\left(Q^{-1}\right)$.

Lemma 4. For an arbitrary integer $s \geq 2$, let $\mathbf{a}=\left(a_{1}, \ldots, a_{s}\right) \in \mathbb{R}^{s}$ so that $1, a_{1}, \ldots, a_{s}$ are linearly independent over $\mathbb{Z}$. Suppose further that there exists a function $\phi: \mathbb{R}^{+} \rightarrow \mathbb{R}^{+}$such that $\phi(t) / t$ increases monotonically and

$$
\|\mathbf{h} \cdot \mathbf{a}\| \geq \frac{1}{\phi\left(|\mathbf{h}|_{\infty}\right)}
$$

for all $\mathbf{h} \in \mathbb{Z}^{s} \backslash\{\mathbf{o}\}$, where $\|\cdot\|$ denotes the distance from the nearest integer. Then for any positive integers $N_{0}$ and $N$, the discrepancy modulo $1 D_{N_{0}, N}(n \mathbf{a})$ of the sequence $(n \mathbf{a})_{n=N_{0}+1}^{N_{0}+N}$ satisfies

$$
D_{N_{0}, N}(n \mathbf{a}) \leq c^{s} s ! \frac{\log N \log \phi^{-1}(N)}{\phi^{-1}(N)},
$$

where $c$ is an absolute constant, $\phi^{-1}$ denotes the inverse function of $\phi$, and $N$ is supposed to be so large that $\phi^{-1}(N) \geq e$.

Proof. This is essentially Theorem 1.80 in the monograph of Drmota and Tichy [4], p. 70, with the dependance on the dimension worked out explicitly.

5. Proof of the Theorem. As already stated, the case of even $\ell$ has been treated in part I of this work [20]. Therefore, we may suppose throughout that $\ell$ is odd. We start from Lemma 3.1 in Zhai [24] which approximates the error term involved by a fractional part sum. Let $U$ be a large real parameter, $u \in[U-1, U+1]$, and put

$$
E(u):=\frac{2^{\ell-2}(\ell-1) !}{r_{1} \cdot \ldots \cdot r_{\ell}} R\left(2 \pi u^{2}\right) .
$$

Then according to Zhai ${ }^{(5)}$ [24], Lemma 3.1, for $\operatorname{arbitrary}^{(6)} \ell \geq 1$,

$$
\begin{aligned}
E(u) & =E^{*}(u)+O\left(u^{2 \ell-1}\right), \\
E^{*}(u) & :=-\sum_{1 \leq m \leq u} m\left(u^{2}-m^{2}\right)^{\ell-1} \psi\left(\frac{u^{2}}{2 m}-\frac{m}{2}-\frac{\ell}{2}\right) .
\end{aligned}
$$

We apply Lemma 1 in the form $-\psi \geq \Sigma_{H}-\Sigma_{H}^{*}$, choosing $H=[U]$. Thus we get

$$
\begin{aligned}
& E^{*}(u) \geq-U+\sum_{1 \leq h \leq U} \sum_{1 \leq m \leq u} m\left(u^{2}-m^{2}\right)^{\ell-1} \times \\
& \times(-1)^{h \ell}\left(\alpha_{h,[U]} \sin \left(2 \pi h\left(\frac{u^{2}}{2 m}-\frac{m}{2}\right)\right)-\beta_{h,[U]} \cos \left(2 \pi h\left(\frac{u^{2}}{2 m}-\frac{m}{2}\right)\right)\right) .
\end{aligned}
$$

(5) In fact, Zhai in his notation tacitly assumes that $r_{1}=\ldots=r_{\ell}=1$, which means no actual loss of generality. We have supplemented the factor $r_{1} \cdot \ldots \cdot r_{\ell}$ in (5.1).

(6) At this stage, we write up the argument for general $\ell$, in order to point out the importance of the condition that $\ell$ is odd later on. 
We split up the range $1 \leq m \leq u$ into dyadic subintervals $\left.\left.\mathcal{M}_{j}=\right] M_{j+1}, M_{j}\right], M_{j}=u 2^{-j}$ for $j=0, \ldots, J$, where $J$ is minimal such that $(U-1) 2^{-J-1}<1$. We thus have to deal with exponential sums

$$
\mathcal{E}_{j}(h, u):=\sum_{m \in \mathcal{M}_{j}} m\left(u^{2}-m^{2}\right)^{\ell-1} e\left(-h\left(\frac{u^{2}}{2 m}-\frac{m}{2}\right)\right) .
$$

We transform them by Lemma 2, with $G(\xi)=\xi\left(u^{2}-\xi^{2}\right)^{\ell-1}, F(\xi)=-h\left(\frac{u^{2}}{2 \xi}-\frac{\xi}{2}\right)$. On each interval $\mathcal{M}_{j}$ the conditions of Lemma 2 are fulfilled with the parameters $X=M_{j}$, $Y=\frac{M_{j}^{3}}{h u^{2}}, Z=M_{j} u^{2 \ell-2}$. By straightforward computations, as in [20], we obtain

$$
\begin{aligned}
\mathcal{E}_{j}(h, u)= & h^{3 / 4} u^{2 \ell-1 / 2} \sum_{k \in F^{\prime}\left(\mathcal{M}_{j}\right)} \frac{(2 k-2 h)^{\ell-1}}{(2 k-h)^{\ell+1 / 4}} e\left(-u \sqrt{h} \sqrt{2 k-h}-\frac{1}{8}\right) \\
& +O\left(u^{2 \ell-3} \frac{M_{j}^{5 / 2}}{h^{1 / 2}}+u^{2 \ell-1} \log u\right) .
\end{aligned}
$$

It is plain to see that the overall contribution of the error terms to (5.3), summed over $j$ and $h$, is $\ll u^{2 \ell-1 / 2}$ : See [20], f. (5.5). Summing up the main terms in (5.4), the total range of $k$ becomes $h=F^{\prime}\left(M_{0}\right)<k \leq F^{\prime}\left(M_{J+1}\right)=\frac{1}{2} h+2^{2 J+1} h=: K_{h, U}$, hence

$$
\begin{aligned}
& \sum_{1 \leq h \leq U} \sum_{1 \leq m \leq u} m\left(u^{2}-m^{2}\right)^{\ell-1}(-1)^{h \ell} \gamma_{h,[U]} e\left(-h\left(\frac{u^{2}}{2 m}-\frac{m}{2}\right)\right)= \\
& =u^{2 \ell-1 / 2} \sum_{h=1}^{[U]}(-1)^{h \ell} \gamma_{h,[U]} h^{3 / 4} \sum_{h<k \leq K_{h, U}} \frac{(2 k-2 h)^{\ell-1}}{(2 k-h)^{\ell+1 / 4}} e\left(-u \sqrt{h} \sqrt{2 k-h}-\frac{1}{8}\right)+ \\
& +O\left(u^{2 \ell-1 / 2}\right),
\end{aligned}
$$

where $\gamma_{h,[U]}$ stands for either $\alpha_{h,[U]}$ or $\beta_{h,[U]}$. Using the real and imaginary part of this result in (5.3), we arrive at

$$
E^{*}(u) \geq u^{2 \ell-1 / 2} S(u, U)-c_{1} u^{2 \ell-1 / 2},
$$

where

$$
\begin{gathered}
S(u, U):=\sum_{(h, k) \in \mathcal{D}(U)} h^{3 / 4} \frac{(2 k-2 h)^{\ell-1}}{(2 k-h)^{\ell+1 / 4}} \times \\
\times(-1)^{h \ell}\left(\alpha_{h,[U]} \sin \left(2 \pi u \sqrt{h} \sqrt{2 k-h}+\frac{\pi}{4}\right)-\beta_{h,[U]} \cos \left(2 \pi u \sqrt{h} \sqrt{2 k-h}+\frac{\pi}{4}\right)\right), \\
\mathcal{D}(U):=\left\{(h, k) \in \mathbb{Z}^{2}: 1 \leq h \leq U, h<k \leq K_{h, U}\right\},
\end{gathered}
$$

and $c_{1}$ is an appropriate positive constant. The next step is to eliminate the majority of the terms of the last double sum. To this end, let $T$ be another large parameter, with 
the constraint that $U \geq T^{2}$. Using Lemma 3, we multiply $S(u, U)$ by the Fejér kernel $\mathcal{F}_{T}(u-U)$ and integrate over $U-1 \leq u \leq U+1$. Thus

$$
\begin{aligned}
& I(T, U):=\int_{U-1}^{U+1} S(u, U) \mathcal{F}_{T}(u-U) \mathrm{d} u=\int_{-1}^{1} S(U+v, U) \mathcal{F}_{T}(v) \mathrm{d} v= \\
& =\sum_{(h, k) \in \mathcal{D}(U), h(2 k-h) \leq T^{2}} h^{3 / 4} \frac{(2 k-2 h)^{\ell-1}}{(2 k-h)^{\ell+1 / 4}}\left(1-\frac{\sqrt{h(2 k-h)}}{T}\right)(-1)^{h \ell} \times \\
& \times\left(\alpha_{h,[U]} \sin \left(2 \pi U \sqrt{h(2 k-h)}+\frac{\pi}{4}\right)-\beta_{h,[U]} \cos \left(2 \pi U \sqrt{h(2 k-h)}+\frac{\pi}{4}\right)\right) \\
& +O\left(\sum_{(h, k) \in \mathcal{D}(U)} h^{-3 / 4} \frac{(2 k-2 h)^{\ell-1}}{(2 k-h)^{\ell+3 / 4}}\right) .
\end{aligned}
$$

The $O$-term here is in fact $O(1)$ : see $[20]$, f. (5.9). Now recall that $(h, k) \in \mathcal{D}(U)$ explicitly means that

$$
1 \leq h \leq U, \quad h<k \leq \frac{1}{2} h+2^{2 J+1} h \asymp U^{2} h,
$$

while $h(2 k-h) \leq T^{2}, k>h$ implies that

$$
h \leq T \leq \sqrt{U} \text { and } \quad k<2 k-h \leq \frac{T^{2}}{h} \leq T^{2} \leq U
$$

Hence the summation condition on the right hand side of (5.7) can be simplified to

$$
0<h(2 k-h) \leq T^{2}, \quad k>h .
$$

For any $h, k$ satisfying (5.8), write $h(2 k-h)=r^{2} q$, with $r$ an integer and $q$ square-free. Now suppose we can choose $U$ so that

$$
\left\|U \sqrt{q}-\frac{1}{2}\right\| \leq \frac{\varepsilon_{0}}{T}
$$

for all square-free $\left.q \in] 1, T^{2}\right]$. Here $\|\cdot\|$ denotes the distance from the nearest integer and $\varepsilon_{0}>0$ is a suitably small constant. Then, for $r^{2} q \leq T^{2}, q>1$,

$$
\sin \left(2 \pi U r \sqrt{q}+\frac{\pi}{4}\right)=\frac{(-1)^{r}}{\sqrt{2}}+O\left(\varepsilon_{0}\right)
$$

By the definitions in Lemma 1, for $h \leq T \leq \sqrt{U}$, it follows that $\alpha_{h,[U]}=\frac{1}{\pi h}+o(1)$, $\beta_{h,[U]} \ll \frac{1}{U}$. Hence, provided that (5.9) is true, for $h(2 k-h)$ not a perfect square,

$$
\begin{aligned}
& \alpha_{h,[U]} \sin \left(2 \pi U \sqrt{h(2 k-h)}+\frac{\pi}{4}\right)-\beta_{h,[U]} \cos \left(2 \pi U \sqrt{h(2 k-h)}+\frac{\pi}{4}\right) \\
& =\frac{1}{h}\left(\frac{(-1)^{h}}{\pi \sqrt{2}}+O\left(\varepsilon_{0}\right)+o(1)\right),
\end{aligned}
$$


where $o(1)$ refers throughout to $U \rightarrow \infty$. (Note that $h, h(2 k-h)=r^{2} q$, and $r$ are of the same parity.) We can use (5.10) in (5.7), appealing at last to the condition that $\ell$ is odd: Then $(-1)^{h \ell}(-1)^{h}=1$, i.e., the alternating factors cancel out. To simplify notation, we put

$$
\theta_{\ell}(n):=\sum_{h(2 k-h)=n, k>h} \frac{h^{1 / 2}}{(2 k-h)^{1 / 2}}\left(1-\frac{h}{2 k-h}\right)^{\ell-1}
$$

With that and (5.10), eq. (5.7) readily yields ${ }^{(7)}$

$$
\begin{aligned}
I(T, U) & \geq c_{2} \sum_{1 \leq n \leq T^{2}} \frac{\theta_{\ell}(n)}{n^{3 / 4}}\left(1-\frac{\sqrt{n}}{T}\right)-c_{3} \\
& \geq c_{4} \sum_{1 \leq n \leq T^{2} / 2} \frac{\theta_{\ell}(n)}{n^{3 / 4}}-c_{3},
\end{aligned}
$$

with certain positive constants $c_{2}, c_{3}, c_{4}$. Further, for $1 \leq n \leq T^{2}$,

$$
\theta_{\ell}(n) \gg \sum_{\substack{h(2 k-h)=n \\ k>2 h}} \frac{h^{1 / 2}}{(2 k-h)^{1 / 2}} \gg \sum_{\substack{h m=n, m>3 h \\ h \equiv m \bmod 2}} \frac{\sqrt{h}}{\sqrt{m}} \gg \sum_{\substack{h m=n, 3 h<m \leq 4 h \\ h \equiv m \bmod 2}} 1 .
$$

Therefore, by (5.12),

$$
\begin{aligned}
& I(T, U)+c_{3} \sum_{\substack{T^{2} / 4 \leq n \leq T^{2} / 2 \\
n \text { odd }}} n^{-3 / 4} \sum_{\substack{h m=n \\
3 h<m \leq 4 h}} 1 \\
& \gg T^{-3 / 2} \sum_{\substack{T^{2} / 4 \leq h m \leq T^{2} / 2 \\
3 h<m \leq 4 h, h, m \text { odd }}} 1 \gg T^{1 / 2} .
\end{aligned}
$$

It remains to ensure the validity of (5.9) and, at the same time, establish a lower bound for $T$ in terms of $U$. To this end we will employ Lemma 4 . Let $q_{1}, \ldots, q_{s}$ be all square-free integers in $\left.] 1, T^{2}\right]$, then obviously $s \asymp T^{2}$. Let further $p_{1}<p_{2}<\ldots<p_{P}$ be all primes $\leq T^{2}$, with $P=\pi\left(T^{2}\right) \asymp T^{2} / \log T$. Consider the field extension $\mathbb{F}=\mathbb{Q}\left(\sqrt{p_{1}}, \ldots, \sqrt{p_{P}}\right)$ and the corresponding Galois group $G=\operatorname{Gal}(\mathbb{F} / \mathbb{Q})$. Since $\chi\left(\sqrt{p_{j}}\right)= \pm \sqrt{p_{j}}$ for $j=$ $1, \ldots, P$ and every $\chi \in G$, obviously $|G|=2^{P}$.

According to Besicovitch's theorem [1], the numbers $1, \sqrt{q_{1}}, \ldots, \sqrt{q_{s}}$ are linearly independent over $\mathbb{Z}$. For any $\mathbf{h}=\left(h_{1}, \ldots, h_{s}\right) \in \mathbb{Z}^{s} \backslash\{\mathbf{o}\}$, let $-h_{0}$ denote the integer nearest to $\mathbf{h} \cdot\left(\sqrt{q_{1}}, \ldots, \sqrt{q_{s}}\right)$, then

$$
\left|\prod_{\chi \in G} \chi\left(h_{0}+h_{1} \sqrt{q_{1}}+\ldots+h_{s} \sqrt{q_{s}}\right)\right| \geq 1
$$

(7) Properly speaking, we are committing here an error concerning the $n$ which are perfect squares. But it is obvious that their contribution to the right-hand side of $(5.12)$ is $\ll \sum m^{-3 / 2+\varepsilon} \ll 1$. 
since the left-hand side is the absolute value of the norm of a nonzero algebraic integer. For any $\chi \in G$,

$$
\left|\chi\left(h_{0}+h_{1} \sqrt{q_{1}}+\ldots+h_{s} \sqrt{q_{s}}\right)\right| \leq 3 s|\mathbf{h}|_{\infty} T .
$$

Hence,

$$
\left|h_{0}+h_{1} \sqrt{q_{1}}+\ldots+h_{s} \sqrt{q_{s}}\right| \geq\left(3 s|\mathbf{h}|_{\infty} T\right)^{-2^{P}} .
$$

Therefore, the requirements of Lemma 4 are fulfilled with $\phi(t)=(3 s t T)^{2^{P}}$. Thus we may conclude that, for $N$ sufficiently large, the discrepancy modulo $1 D_{N}$ of the sequence $\left(n \sqrt{q_{1}}, \ldots, n \sqrt{q_{s}}\right)_{n=N+1}^{2 N}$ satisfies

$$
D_{N} \leq c^{s} s ! \frac{\log N \log \phi^{-1}(N)}{\phi^{-1}(N)} \ll c^{s} s ! N^{-2^{-P}}(\log N)^{2},
$$

since $\phi^{-1}(N)=N^{2^{-P}}(3 s T)^{-1}$. Now, with (5.9) in the back of mind, let $\mathcal{C}$ denote the $s$-dimensional cube $\left[\frac{1}{2}-\frac{\varepsilon_{0}}{T}, \frac{1}{2}+\frac{\varepsilon_{0}}{T}\right]^{s}$. Writing $\langle\cdot\rangle$ for the fractional part, we may thus conclude that

$$
\begin{aligned}
& \#\left\{n \in \mathbb{Z}: N<n \leq 2 N,\left(\left\langle n \sqrt{q_{1}}\right\rangle, \ldots,\left\langle n \sqrt{q_{s}}\right\rangle\right) \in \mathcal{C}\right\} \\
& \geq N \operatorname{vol}(\mathcal{C})-N D_{N} \geq N\left(\left(2 \varepsilon_{0}\right)^{s} T^{-s}-c_{5}^{s} s ! N^{-2^{-P}}(\log N)^{2}\right) \\
& \geq N\left(\left(c_{6} T\right)^{-c_{7} T^{2}}-\left(c_{8} T\right)^{c_{9} T^{2}} N^{-2^{-P}}(\log N)^{2}\right),
\end{aligned}
$$

using Stirling's formula and $s \asymp T^{2}$. Here $c_{j}$ are throughout absolute positive constants. A short calculation shows that the large bracket is certainly $>0$ if we choose

$$
N=N^{*}(T):=\left[\exp \left(\exp \left(c_{10} T^{2} / \log T\right)\right)\right],
$$

with $c_{10}$ sufficiently large. Therefore, for arbitrary $T$ there exists at least one integer $\left.U=n \in] N^{*}(T), 2 N^{*}(T)\right]$ for which $\left(\left\langle U \sqrt{q_{1}}\right\rangle, \ldots,\left\langle U \sqrt{q_{s}}\right\rangle\right) \in \mathcal{C}$, which means that (5.9) is fulfilled. Further, (5.14) implies that

$$
T \asymp\left(\log _{2} U \log _{3} U\right)^{1 / 2} .
$$

Thus (5.13) yields

$$
I(T, U) \gg\left(\log _{2} U \log _{3} U\right)^{1 / 4} .
$$

On the other hand, it follows from the definition of $I(T, U)$ that

$$
I(T, U) \leq\left(\sup _{U-1 \leq u \leq U+1} S(u, U)\right) \int_{-1}^{1} \mathcal{F}_{T}(v) \mathrm{d} v \leq \sup _{U-1 \leq u \leq U+1} S(u, U) .
$$

This implies that there exists a value $u^{*} \in[U-1, U+1]$ for which

$$
S\left(u^{*}, U\right) \gg\left(\log _{2} u^{*} \log _{3} u^{*}\right)^{1 / 4} .
$$


It remains to recall that if $T$ runs through an unbounded sequence of positive reals, by construction $^{(8)}$ so do $U$ and $u^{*}$. Therefore, (5.1), (5.2), (5.6) and (5.17) together complete the proof of our theorem.

\section{References}

[1] A.S. Besicovitch, On the linear independence of fractional powers of integers, J. London Math. Soc. 15, 3-6 (1940).

[2] K. Corrádi and I. Kátai, A comment on K. S. Gangadharan's paper "Two classical lattice point problems" (Hungarian), Magyar Tud. Akad. mat. fiz. Oszt. Közl. 17, 89-97 (1967).

[3] H. Cramér, Über zwei Sätze von Herrn G.H. Hardy, Math. Z. 15, 201-210 (1922).

[4] M. Drmota and R.F. Tichy, Sequences, discrepancies, and applications, Lecture Notes in Math. 1651, Springer, Berlin, 1997.

[5] K.S. Gangadharan, Two classical lattice point problems, Proc. Cambridge Phil. Soc. 57, 699-721 (1961).

[6] C.S. Gordon and E.N. Wilson, The spectrum of the Laplacian on Riemannian Heisenberg manifolds, Michigan Math. J. 33, 253-271 (1986).

[7] S.W. Graham and G. Kolesnik, Van der Corput's method of exponential sums, Cambridge 1991.

[8] J.L. Hafner, New omega results for two classical lattice point problems, Invent. Math. 63, 181-186 (1981).

[9] G.H. Hardy, On the expression of a number as the sum of two squares, Quart. J. Math. 46, 263-283 (1915).

[10] L. Hörmander, The spectral function of an elliptic operator, Acta Math. 121, 193-218 (1968).

[11] M.N. Huxley, Area, lattice points, and exponential sums, LMS Monographs, New Ser. 13, Oxford 1996.

[12] M.N. Huxley, Exponential sums and lattice points III. Proc. London Math. Soc. (3) 87, 591-609 (2003).

[13] A. Ivić, E. Krätzel, M. Kühleitner, and W.G. Nowak, Lattice points in large regions and related arithmetic functions: Recent developments in a very classic topic. Proceedings Conf. on Elementary and Analytic Number Theory ELAZ'04, held in Mainz, May 24-28, W. Schwarz and J. Steuding eds., Franz Steiner Verlag 2006, pp. 89-128.

[14] H. Iwaniec, E. Kowalski, Analytic Number Theory, AMS Coll.Publ. 53. Providence, R.I., 2004.

[15] M. Khosravi, Spectral statistics for Heisenberg manifolds, Ph.D. thesis, McGill U. 2005.

[16] M. Khosravi and Y.N. Petridis, The remainder in Weyl's law for $n$-dimensional Heisenberg manifolds, Proc. AMS 133/12, 3561-3571 (2005).

[17] M. Khosravi and J.A. Toth, Cramer's formula for Heisenberg manifolds, Ann. de l'institut Fourier $\mathbf{5 5}, 2489-2520$ (2005).

[18] E. Krätzel, Lattice points. Berlin 1988.

[19] E. Krätzel, Analytische Funktionen in der Zahlentheorie. Stuttgart-Leipzig-Wiesbaden 2000.

[20] W.G. Nowak, A lower bound for the error term in Weyl's law for certain Heisenberg manifolds, submitted for publication. Available online at: http://arxiv.org/PS_cache/arxiv/pdf/0809/0809.3924v1.pdf

[21] Y.N. Petridis and J.A. Toth, The remainder in Weyl's law for Heisenberg manifolds, J. Diff. Geom. 60, 455-483 (2002).

(8) Recall the ultimate order in the choice of the parameters: For $T$ an independent large real variable, $N^{*}(T)$ is defined by (5.14), then $U$ is picked from $\left.] N^{*}(T), 2 N^{*}(T)\right]$, finally $u^{*}$ from $[U-1, U+1]$. The condition $U \geq T^{2}$ needed earlier in the argument is amply satisfied in view of (5.15). 
[22] K. Soundararajan, Omega results for the divisor and circle problems. Int. Math. Res. Not. 36, 1987-1998 (2003).

[23] J.D. Vaaler, Some extremal problems in Fourier analysis, Bull. Amer. Math. Soc. 12, 183-216 (1985). [24] W. Zhai, On the error term in Weyl's law for the Heisenberg manifolds, Acta Arithm. 134, 219-257 (2008).

\author{
Institute of Mathematics \\ Department of Integrative Biology \\ Universität für Bodenkultur Wien \\ Gregor Mendel-Straße 33 \\ 1180 Wien, Österreich \\ E-mail: nowak@boku.ac.at
}

